\title{
Development of Offshore Platform Stress Monitoring System based on Internet of Things Technology
}

\author{
YongShun $\mathrm{Wu}^{1}$, Demin $\mathrm{Chen}^{2}$, Zhaodan $\mathrm{Xia}^{1}$ and Sujun Yang ${ }^{13 *}$ \\ ${ }^{1}$ Marine Design \& Research Institute of China, 200011, China \\ ${ }^{2}$ Beijing Beetech Inc, 100085, China \\ ${ }^{3}$ Science and Technology on Water Jet Propulsion Laboratory, 200011, China
}

\begin{abstract}
This paper mainly analyzes the main external load types of offshore platforms and the current status of offshore platform health monitoring technology. On this basis, a platform health monitoring system which is consist of a wireless sensor network and a satellite communication system is an typical application of Internet of Things Technology in the field of offshore platform monitoring. This paper has a detailed discussion of the overall system and key equipment. It has certain reference significance to engineers and technicians in related fields.
\end{abstract}

\section{Introduction}

With oil extraction develops into the ocean, the number of offshore platforms doubles, and appropriate design methods ensure that the structure resists damage caused by unpredictable loads, but damage is unavoidable during service of the offshore platform structure ${ }^{[1]}$. The structural health monitoring technology is actually the inverse problem of the traditional structural dynamics problem. The technology of structural damage is comprehensively evaluated by real-time and nondestructive monitoring of the structure. The only way to ensure human life and reduce property damage is to diagnose structural damage and repair it in a timely manner.

It can be seen that the problem of improving the reliability of the structure and equipment of the offshore platform and ensuring the safety of offshore operations is becoming increasingly prominent. The quality evaluation of the new platform, the estimation of the residual life of the old platform and the structural safety guarantee of the in-service platform will become increasingly prominent problems. The health monitoring of the offshore platform structure has become an important issue that cannot be delayed.

The research on the health monitoring of offshore platforms began in the 1970s, and the research field mainly involved cracks, corrosion and monitoring of structural stresses and deformations. The existing offshore platform health monitoring is mainly concentrated in the following aspects; the first aspect is the monitoring of environmental loads, the second aspect is the monitoring of corrosion and cracks, and the third is the vibration-based health monitoring. Although there are many vibration damage identification methods, due to the complex structure and environment of the offshore platform, the effective and accurate application has encountered great difficulties. It is difficult to measure the artificial excitation of the offshore platform, and the environmental excitation is difficult to measure. It is impossible for the platform monitoring system to monitor every component. In addition, people have limited understanding and experience of the response of the offshore platform under the environmental load excitation, which leads to the monitoring of individual key points has omission, modal parameters can not be accurately identified, which has become a bottleneck for the health monitoring of offshore platform structures ${ }^{[2,3]}$.

\section{System structure design and working principle}

The offshore platform health monitoring system structure is shown in Figure1, which is mainly composed of two parts. The first part is the wireless sensor network that senses the physical parameters of the platform, such as vibration, stress, temperature, displacement, etc, which is the most important for the system. During the physical parameters, the stress parameters mainly affect the safety of the platform, which the paper introduces. If other physical quantities need to be monitored, proper types of sensors can be directly selected based on the monitoring platform. The second part is transferring wireless sensor network data forward to the external network of the remote monitoring host; the system involved in this paper forwards the data to the onshore monitoring host through satellite (Beidou satellite or maritime satellite). During this system, wireless stress acquisition instruments and sensors play an important rule.

The operation program of the offshore platform structure health monitoring system is that the signal

*Corresponding author's e-mail: sujuny@163.com 
picked up by the sensor measurement system is first obtained by the data acquisition unit, and then the collected signal is converted into a digital signal and transmitted to the data forwarding gateway through the short-range micro-power wireless communication module, and the data is forwarded. The gateway transmits data to the monitoring computer system by means of an external communication system (such as the Beidou satellite communication system) to complete post-processing, archiving, display and storage of the data. Finally, the high-performance computer system completes the evaluation of the structural health monitoring system and provides the platform reports in real time during the whole work process, and alarms the abnormal state of the offshore platform structure, evaluates the safety and practicability of the offshore platform in real time, provides a direct basis for the periodic inspection of the offshore platform, and submits monitoring and health status evaluation report.

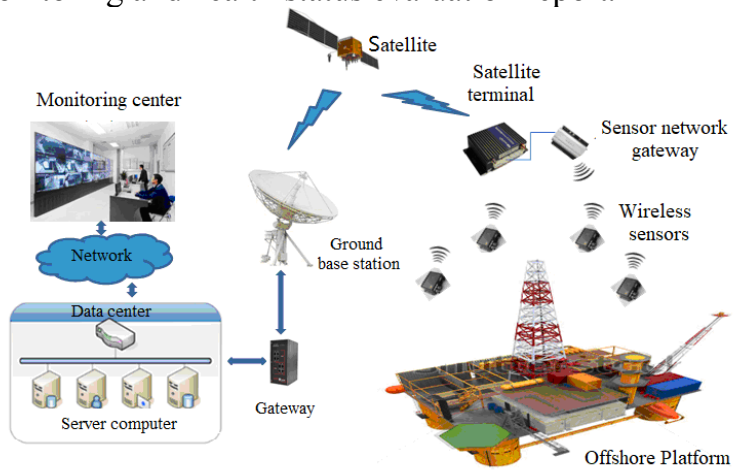

Figure 1 System structure and composing

\section{The realization of wireless stress data acquisition}

The realization of wireless stress data acquisition is shown in Figure2, which is developed based on the wireless sensor network platform. Multiple distributed wireless stress monitoring sensors are distributed to realize multi-measurement and multi-data synchronous measurement. The data collected by the plurality of stress sensors is aggregated in the wireless gateway through the wireless sensor network, and the wireless gateway forwards the received multi-sensor data to the local monitoring host or the external network (including the Beidou satellite system, etc.). The remote monitoring host stores, displays, and processes data collected by the

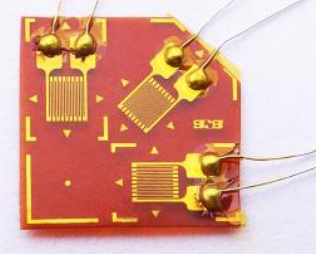

Figure 3 Three dimension micro-strain Figure4 Single dimension micro-strain

\subsection{Wireless stress acquisition node design principle}

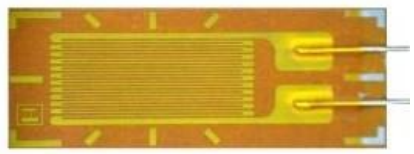

wireless stress collection node by using an external network.

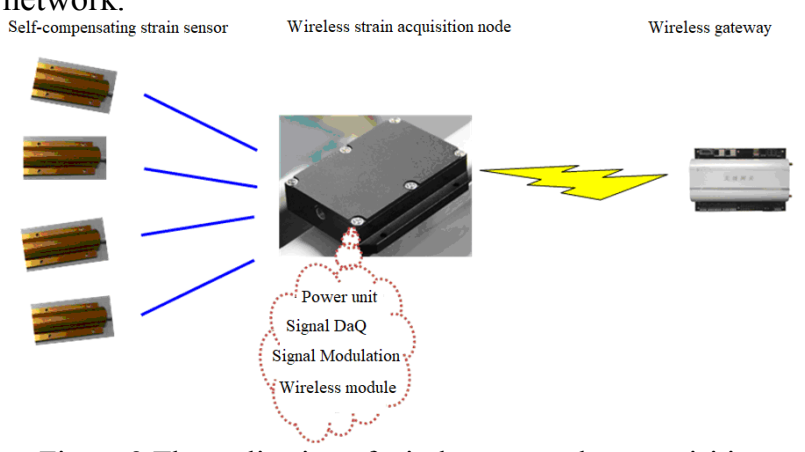

Figure 2 The realization of wireless stress data acquisition

\subsection{Self-compensating micro-strain sensor}

Wireless stress sensors require an external strain sensor to measure the structural stress of the offshore platform. It can be seen from the above discussion that the structure of the offshore platform is subjected to various loads and the force is relatively complicated. Combined with the characteristics of the field application environment, this paper designs a self-compensating strain sensor. The sensor has two types; one is a strain flower with three-dimension angle of 90 degrees. Another one is a two-wire monolithic, according to the characteristics of the measuring point, the strain flower or single piece is arranged. For each channel of the wireless strain sensor, each channel is measured with $1 / 4$ bridge, and three dimension strain requires three wireless strain sensor acquisition channels (shown in Figure3 and 4).

Through the study of the measurement bridge and strain gauge, it can be known that the conventional $1 / 4$ bridge single dimension strain gauge has the influence of temperature and wire resistance. In order to achieve better long-term stress measurement stability, the bridge compensation method is adopted in this paper.

The specific embodiment is as follows: one strain gauge is attached to the test piece, and the other strain gauge is pasted on the compensation block. For each measurement channel, the compensation principle is the same whether it is a strain flower or a 2-wire monolithic. One piece is tested, one piece is temperature and line resistance compensated, and two strain gauges form a half bridge.

The wireless strain detection system is mainly composed of signal modulation and acquisition circuit, CPU unit, wireless communication module and power supply unit. 
Figure5 is a block diagram of the wireless strain detection sensor.

When measuring with a strain gauge, the strain gauge is stuck on the object to be tested. When the structure of the object is stressed, deformation will occur, and the sensitive grid of the strain gauge will be elongated or shortened, and the resistance value will change accordingly. By measuring the bridge, the change in resistance value is converted to a change in voltage or current.

The data collected by the sensor can be stored in the node in real time, and can also be transmitted to the host computer in real time, thereby ensuring the security and accuracy of the collected data. The node's wireless transmission rate can reach 250KBPS, and its communication distance can reach 300 meters. There are power management hardware and software inside the node. When data is continued to be transmitted, the node consumes only a few mA of power. The node has built-in rechargeable battery, which can be used for more than half a year once time. The node supports external I/O trigger acquisition control mode, and accesses high level signal through external interface for control. The node has USB interface, and the user can use the USB interface to charge the node, and the data in the memory of the node can be quickly downloaded.

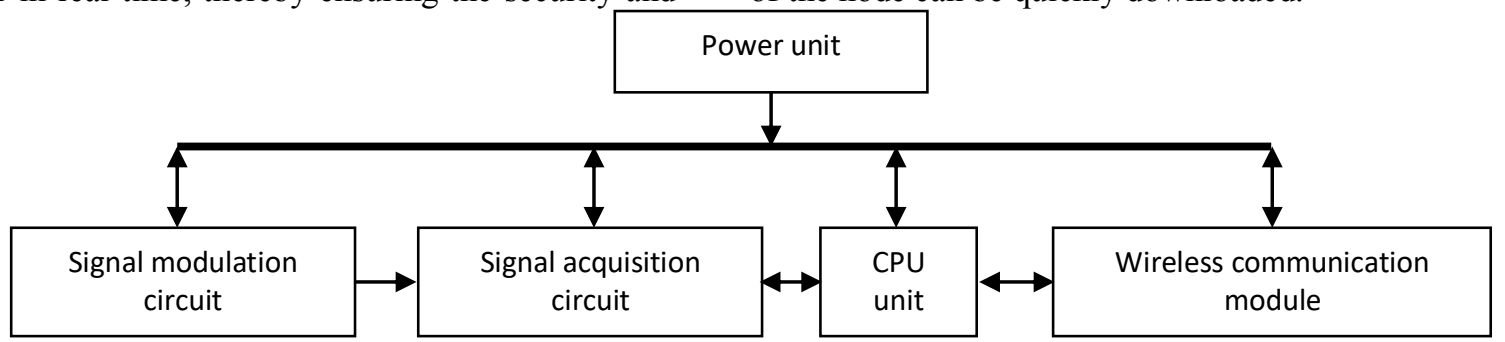

Figure 5 Block diagram of the wireless strain acquisition node

\subsection{The micro strain sensor and its signal line protection method}

The environment of the offshore platform is complex and affected by environmental factors such as seawater, high temperature and low temperature. The protection of the strain gauge is an extremely important link in the success of the test. The main purpose of the protection is to prevent seawater from invading the strain gauge, destroying the electrical properties of the strain gauge and the chemical stability of the binder, and cannot accurately reflect the strain signal of the structure. In this paper, the test piece and the compensation piece are divided into a protective box. The basic process structure is shown in Figure6 and 7. The test strain gauge is pasted on the test piece, and the test piece is covered with special protective glue. When the protective glue is dry, the protective glue is covered on it again, and the protective glue is applied on the surface to ensure that the strain gauge is completely covered, and the protective layer is completely covered. Above the protective layer, apply soft rubber with relatively large elasticity, the glue layer is at least $2 \mathrm{~mm}$ thick, then place the compensation piece on it, and the compensation piece is pasted with the compensation strain gauge. The protection process is consistent with the test strain gauge, and then the stainless steel protective cover is fastened to a fixed strain gauge. On a fixed strain gauge, the stainless steel protective shell is surrounded by an extended edge and welded to the platform structure. After that, brush the seawater proof paint on the stainless steel casing and paint it multiple times.

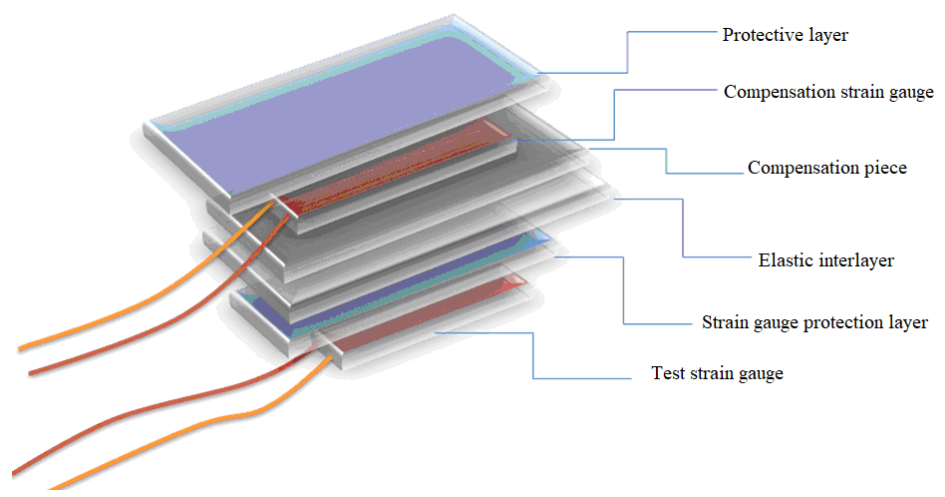

Figure 6 Strain protection process

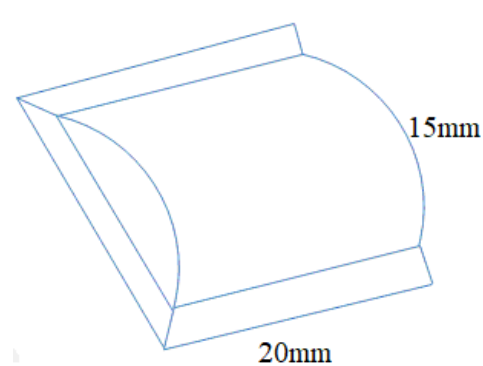

Figure 7 Protective casing
The measuring wire of the strain gauge is more than ten meters long. This paper uses $<22 \times 3$ stainless steel pipe, which is welded to the outer wall of the main pipe and the inner wall of the pile in advance, and is connected with the strain gauge protection box, so that the wires of the underwater part are all passed through the protection pipe to the top of the pile and the top of the jacket. The wire protection steel pipe is welded to the pile or beam in advance. The strain gauge wire inside is made of tensile and waterproof wires, and the protective 
glue is applied to the joint of the wire and the strain gauge to prevent seawater treatment. The wire is not tightened in the protective pipe. The balance is to prevent the wire skin from rupturing and the insulation to be damaged. The protective pipe is coated with a multilayer anti-seepage and anti-corrosive paint.

\subsection{Features of wireless stress acquisition}

The wireless stress sensor designed in this paper is a new data acquisition system based on 802.15.4 protocol. It is an upgraded version of the Beetech standard SG404 wireless strain sensor. It is very simple to use, and it can directly suck the node with the magnet attached to the object to be tested. The sensor transmits wireless digital signals, which eliminates the noise interference caused by the long cable transmission traditionally used, so that the entire measurement system has high measurement accuracy and strong anti-interference ability. The sensor has four independent stress acquisition channels, each with built-in independent high-precision bridge resistors and amplification conditioning circuitry to support voltage excitation. At the same time, this node can easily realize automatic switching of $1 / 4$ bridge, $1 / 2$ bridge and full bridge measurement mode through the host computer software, which is compatible with various types of bridge sensors.

The sensor has a large sampling rate setting range, which can realize static stress data acquisition and dynamic stress data acquisition, as well as short-term structural stress testing and long-term structural stress health monitoring. When the sampling rate is less than $1 \mathrm{sps}$, after the setting is successful, click "Start Acquisition" to enter the monitoring state. At this time, the power consumption is about $1 / 500$ of the normal working time. When the sampling rate is a few $\mathrm{Hz}$, static data acquisition can be done. The sensor is supporting a variety of sampling rates available: $3600 \mathrm{~s}, 1800 \mathrm{~s}, 600 \mathrm{~s}$, $30 \mathrm{~s}, 60 \mathrm{~s}, 10 \mathrm{~s}, 5 \mathrm{~s}, 1 \mathrm{hz}, 10 \mathrm{hz}, 100 \mathrm{hz}, 200 \mathrm{hz}, 500 \mathrm{hz}$, $1000 \mathrm{hz}$.

\section{System function}

The offshore platform structure health monitoring and safety evaluation system's overall goal is to monitor the performance of the offshore platform and evaluate the working conditions of the offshore platform by measuring certain information reflecting the environmental incentives and structural response status of the offshore platform, and to provide a scientific basis for the maintenance of offshore platform. Unlike traditional offshore platform detection methods (including numerous non-destructive testing techniques), offshore platform structural health monitoring and safety assessment systems focus on diagnosing conditions and environmental factors that may cause structural damage or disasters, and assessing signs and trends of structural performance degradation, in order to take timely maintenance measures.

The traditional detection method focuses on checking the existence of damage after the damage occurs, and adopts the means of repair and reinforcement. Therefore, the main functions of the offshore platform structural health monitoring and safety assessment system include: confirming the actual performance of the offshore platform, ensuring that the design goals are achieved; increasing the safety of the offshore platform structure to ensure that the offshore platform can be used safely for a long time; serving the offshore platform construction monitoring and integrated management system, reducing the number of manual inspections in non-key areas, assessing safety during accidents and afterwards, assisting and improving the detection methods of offshore platforms, providing a basis for maintenance decisions of offshore platforms, developing offshore platform structure health monitoring and evaluation techniques and methods.

\section{Conclusions}

The main contents and objectives of the monitoring program include: long-term real-time monitoring of the force and deformation of the cantilever beam structure of the platform, real-time evaluation and early warning of the safety of the cantilever beam structure of the platform; establishing a health file of the cantilever beam structure of the platform in order to provide a reliable basis for the daily operation, working conditions and maintenance of the cantilever beam of the platform; the current state of force of the cantilever beam structure of the platform can be presented in real time through the vivid display of the image, which provides a basis for the safe operation of the cantilever beam of the platform.

The offshore platform monitoring system designed in this paper can be transplanted to the marine ship monitoring field to realize the ship IOT monitoring system. The offshore platform health monitoring system integrates knowledge in many fields such as modern sensing technology, network communication technology, signal processing and analysis, data management methods, computer vision, knowledge mining, prediction technology, structural analysis theory and decision theory. The connotation of the offshore platform monitoring testing field is expanded and the forecasting evaluation is improved.

\section{References}

1. Lin Changgang. Study on wind load and storm environment map of jack-up offshore platform [D]. DalianUniversity of Technology. 2013.

2. Yang Guoan, Gai Huiming. Research on New Methods for Structural Health Monitoring of Offshore Platforms [J]. Research Design, 2007 (03): $20 \sim 23$.

3. Liu Ling, Lu Jianhui, Li Yuhui. Research methods and progress of health monitoring for offshore oil platforms [J]. Petroleum Engineering Construction, 2005 (01) $17 \sim 21$. 
4. Chen Demin. Sensor World of Fan Stress Monitoring System Based on Wireless Sensor Network [J]. 2011 (02): $21 \sim 23$.

5. NieBinglin. New Progress in Underwater Detection and Monitoring Technology at Home and Abroad [J]. China Offshore Platform, 2005, 20 (6): $43 \sim 45$.
6. Hou Qiang, Zhang Yantao, Liu Yongqing. In-situ dynamic stress test of offshore platform [J]. Coastal Engineering. 2000, 3 (19): $20 \sim 26$. 